\title{
Sub-Molecular Potential Profiling Across Organic Monolayers
}

\author{
Neta Filip-Granit ${ }^{\mathrm{a}, \mathrm{b}}$, Milko E. van der Boom ${ }^{\mathrm{a}}$, Roie Yerushalmi ${ }^{\mathrm{c}}$, Avigdor Scherz ${ }^{\mathrm{b}}$ and \\ Hagai Cohen ${ }^{* d}$ \\ a- Department of Organic Chemistry, The Weizmann Institute of Science, Rehovot, \\ 76100, Israel; \\ b- Department of Plant Sciences, The Weizmann Institute of Science, Rehovot, 76100, \\ Israel; \\ c- Electrical Engineering and Computer Science, University of California, Berkeley, CA; \\ d- Department of Chemical Research Support, The Weizmann Institute of Science, \\ Rehovot, 76100, Israel
}

\section{Supplementary Material}

\section{A. The Coupling layers}

The XPS and CREM analyses of the coupling layers, $\mathrm{X}=\mathrm{I}$ and $\mathrm{X}=\mathrm{Cl}$, Fig. $1 \mathrm{~d}$ in the paper, indicate the evolution of beam induced damage, on which we provide here further details. For characterizations with additional methods, the reader is referred to Ref. 20 of the manuscript.

Measurements were performed on a slightly modified Kratos AXIS-HS spectrometer, using monochromatized $\mathrm{Al}\left(\mathrm{k}_{\alpha}\right)$ radiation.at low x-ray power, $5 \mathrm{~mA}$ x 15 $\mathrm{kV}$. Low electron flood gun (eFG) current densities were used in all cases, <100 nA with an effective spot diameter of 3-4 $\mathrm{mm}$.

The dominant damage process observed under exposure to irradiation is expressed in a gradual removal of halogen atoms. Slight changes in the carbon concentration (an order of magnitude smaller) are also observed, while no change in the Si and the substrate signal intensities have been detected, except for their trivial increase upon removal of overlayer atoms. Accordingly, the effective thickness of the layer, as derived from attenuation considerations, suffers less than 10\% decrease along 20 hours of irradiation (x-rays plus eFG electrons). 
Initially, the desorption of $\mathrm{Cl}$ atoms from the $\mathrm{X}=\mathrm{Cl}$ layers is relatively rapid, but it slows down and practically vanishes after a few hours of irradiation. No chemical shift at the $\mathrm{Cl}(2 \mathrm{p})$ line and no indication for $\mathrm{Cl}$ migration towards the substrate could be observed, while slight ( 100 meV, CREM derived) collective electrostatic drifts clearly took place during the process of halogen dilution (not shown). Fig. S1 demonstrates the halogen intensity decrease with time (t) and the stabilization at $\sim 70 \%$ of the initial concentration.

A similar behavior is found in the $\mathrm{X}=\mathrm{I}$ samples, with faster kinetics and a residual $\mathrm{Cl}$ concentration, where the $\mathrm{Cl} / \mathrm{I}$ ratio is roughly retained during the halogen desorption. A reduced character of iodine signal emerges under irradiation (Fig. S2), becoming eventually the dominant state. Stabilization is practically realized in these samples at about $65 \%$ of the initial iodine concentration (subjected to total $11 \%$ of further loss during 32 hours).

At minimal exposure time, our XPS analyses yield atomic concentrations, which are in good agreement with the theoretical expectations. Table S1 presents characteristic numbers, where $\mathrm{X}$ stands for the total halogen signal; $\mathrm{I}_{\mathrm{red}} / \mathrm{I}$ is the relative portion of reduced signal in the iodine line; and $d$ is the effective layer thickness, given in angstroms. The theoretical values in Table S1 account for electron attenuation corrections, assuming ideally organized, vertically oriented molecules, with photoelectron inelastic mean free path of $\lambda=33 \mathrm{~A}$. It is stressed that besides diluting the halogen atoms under irradiation, the monolayer structure is basically retained, in particular its vertical profile.

\section{B. The bi-layers:}

Binding the $\mathrm{BChl}$ molecules to the $\mathrm{X}=\mathrm{Cl}$ coupling layer has not been proven successful. On the other hand, with the $\mathrm{X}=\mathrm{I}$ coupling, efficient formation of a second layer has been achieved, exhibiting saturation characteristics at increasing adsorption 
times. The inset to Fig. 3b illustrates the expected binding site, where a thiol group in the $\mathrm{BChl}$ derivative replaces the iodine of the coupling layer. Molecular cross sections would suggest that each BChl molecule 'covers' about 11-12 coupling molecules. Experimentally, based on the XPS atomic ratios, Table S2, the effective BChl coverage is thus estimated to be $40-80 \%$. This estimation depends on unknown structural parameters, for example via the electron attenuation corrections, which are sensitive to the detailed 2D density across the (non uniform) BChl cross-section. Consequently, broad uncertainty should be considered for the coverage estimations.

Integrating the overall characterization data, we believe that $\mathrm{BChl}$ coverage of about 50\% should fit the CREM studied bi-layers, with extra carbon signal attributed to adsorbed solvent molecules. Independent characterization of the system indicates that the BChl molecules are tilted from the surface normal, the overall bi-layer thickness being 21 $\AA$ (Ref 20), with no close proximity of halogen atoms to the macro-cycle units. The S signal, which is relatively noisy, is in a reasonable agreement with the $\mathrm{N}$ concentration (see Table S2), however showing in some samples an extra signal of oxidized sulfur. Note that the weak sulfur signal could not be used for fine CREM analyses. The total amount of layer interface signals $(\mathrm{X}+\mathrm{S})$ is much smaller than that of the $\mathrm{Si}$, indicating possible replacement of the halogen atoms, mainly iodine, e.g. by $\mathrm{H}$ or $\mathrm{OH}$ species, suspected to take place during the washing procedures. This selective replacement is also expressed in a relatively high $\mathrm{Cl} / \mathrm{I}$ concentration ratio, as compared to our findings at the $\mathrm{X}=\mathrm{I}$ monolayers before the $\mathrm{BChl}$ adsorption.

The bilayer system generally exhibits high stability under the $\mathrm{x}$ ray plus eFG irradiation, and a vertically well-defined arrangement, both features useful for the CREM analyses. 
Tables:

Table S1: XPS analytical data corresponding to minimal exposure times. X stands for the total concentration of halogens $\left(\mathrm{Cl}\right.$ in the first sample and $\mathrm{I}+\mathrm{Cl}$ in the second). $\mathrm{I}_{\mathrm{red}}$ is the reduced part in the iodine signal. Attenuation corrections have been taken into account in the theoretical values.

\begin{tabular}{|c|c|c|c|c|}
\hline Sample & $\mathbf{C} / \mathrm{Si}$ & $\mathrm{X} / \mathrm{Si}$ & $\mathrm{I} / \mathrm{X}$ & $\mathrm{I}_{\text {red }} / \mathbf{I}$ \\
\hline $\mathrm{X}=\mathrm{Cl}$ & $\mathbf{7 . 6}$ & $\mathbf{0 . 9 5}$ & $\mathbf{0}$ & \\
\hline $\mathrm{X}=\mathbf{I}$ & $\leq \mathbf{6 . 9}$ & $\leq \mathbf{0 . 9 8}$ & $\mathbf{0 . 6 5}$ & $\mathbf{0 . 1 - 0 . 3}$ \\
\hline Theory & $\mathbf{7 . 8}$ & $\mathbf{1 . 2 3}$ & & \\
\hline
\end{tabular}

Table S2: Bi-layer analytical data, where $\mathrm{X}$ is the total concentration of halogens $(\mathrm{I}+\mathrm{Cl})$. Pre-assumptions on the density of atoms at the top layer were required for the attenuation corrections, thus reducing the accuracy of the theoretical values.

\begin{tabular}{|c|c|c|c|c|}
\hline & $\mathbf{N} / \mathbf{S i}$ & $\mathbf{N} / \mathbf{S}$ & $\mathbf{C} / \mathbf{S i}$ & $(\mathbf{X + S}) / \mathbf{S i}$ \\
\hline Experiment & 0.2 & 5.7 & $10.5-18.0$ & 0.5 \\
\hline $\begin{array}{c}\text { Theory for } \\
\text { 40\% coverage }\end{array}$ & 0.22 & 6.3 & 10.0 & 1.23 \\
\hline $\begin{array}{c}\text { Theory for } \\
\mathbf{1 0 0 \%} \text { coverage }\end{array}$ & 0.6 & 6.3 & 16.9 & 1.23 \\
\hline
\end{tabular}


Figures:

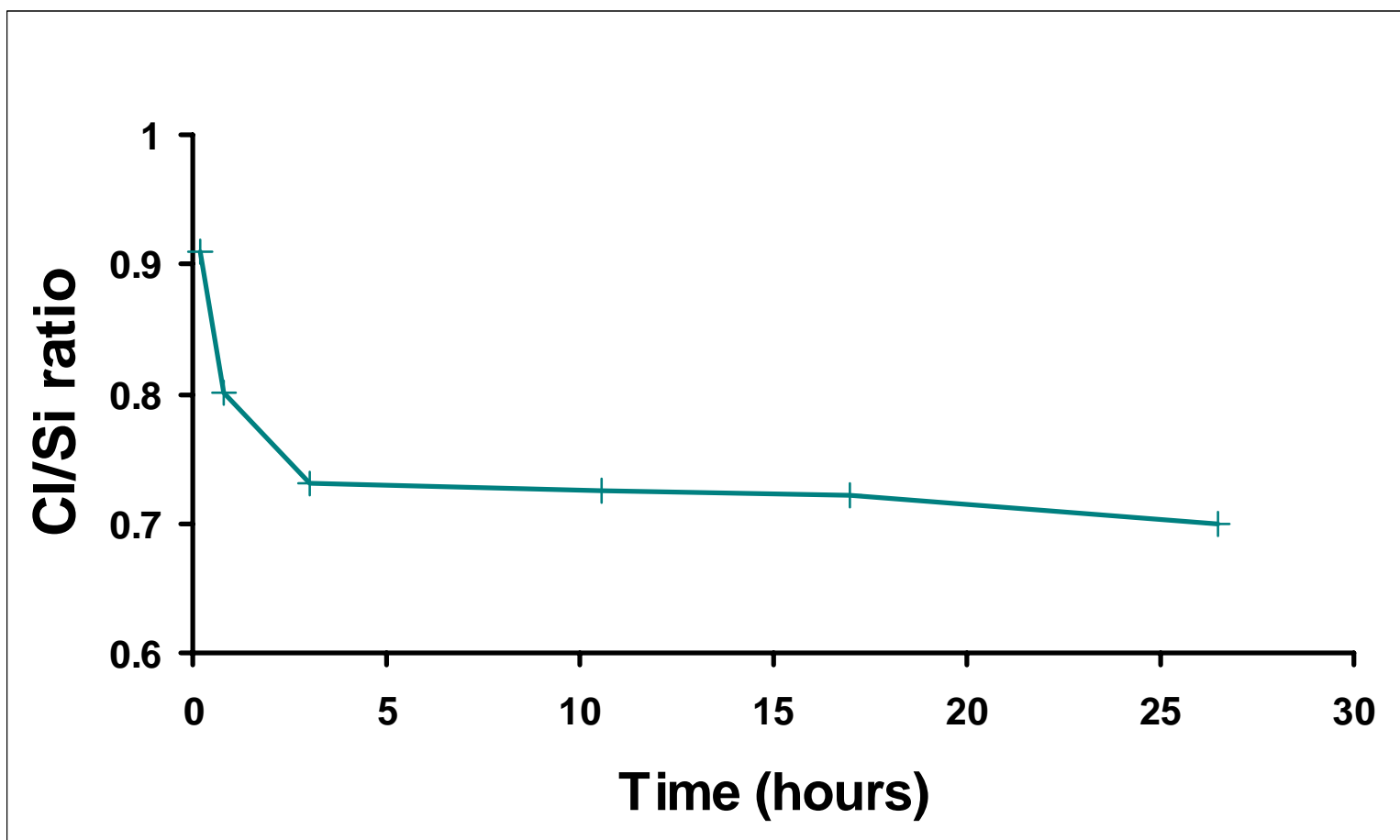

Fig. S1: The evolution in $\mathrm{Cl}$ concentration, normalized to the (stable) Si signal, as a function of exposure time. 


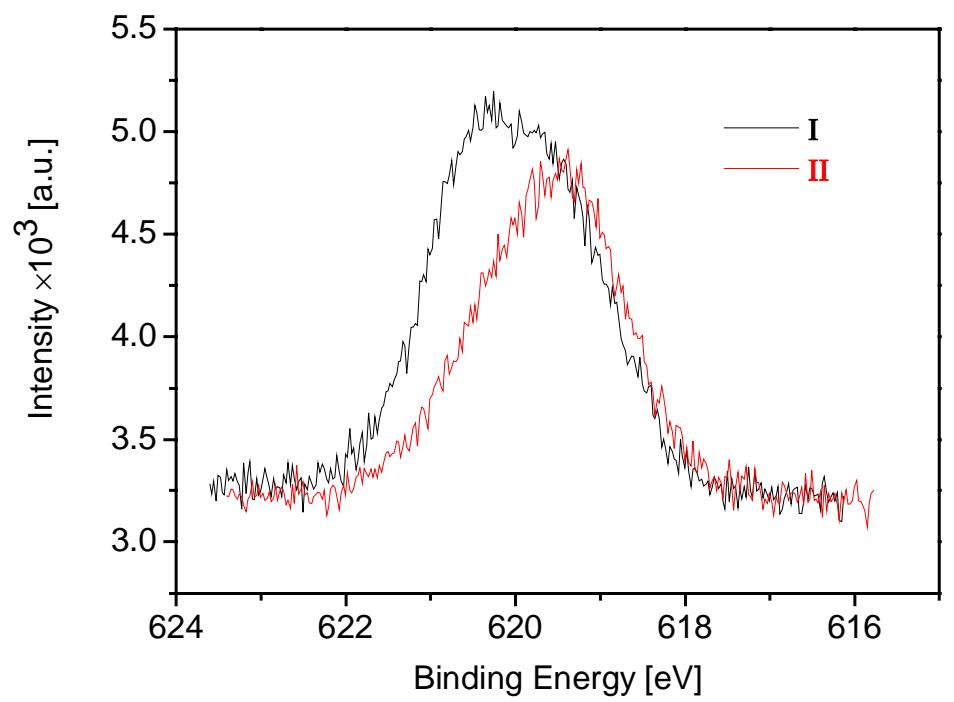

Fig. S2: The iodine $3 d_{5 / 2}$ signal at two early stages of the experiment: (I) after 1.5 hours irradiation and (II) after 2.5 hours. Note the removal of the high binding energy state, while a low binding energy component evolves. Our experimental data do not provide a fully conclusive answer for the limit of zero exposure time, whether it consists of a single iodine state only (at the higher binding energy), or includes already a small portion of reduced iodine. 